\title{
The Synthesis of Composite Particles in Molten Salts
}

\author{
Ruisong Yang*, Lishan Cui and Yanjun Zheng \\ Department of Materials Science and Engineering, University of Petroleum, Beijing, 102249, P. R. China
}

A novel process for synthesizing composite particles, named the high temperature molten salts method, is discussed in this paper. The molten salts are a reaction medium that do not take part in the chemical reaction and can be easily dissolved by water washing. By this method, composite particles were prepared in molten salts at $680-850^{\circ} \mathrm{C}$. The heat released from the chemical reaction was found playing an important role to obtain the desired composite particles. The reverse martensitic transformation of the NiTi particles is confirmed in these composite particles by differential scanning calorimetry (DSC).

(Received August 22, 2005; Accepted September 26, 2005; Published March 15, 2006)

Keywords: nickel titanium particles, molten salts, martensitic transformation

\section{Introduction}

Powder materials can be synthesized by a number of techniques by starting from the vapor phase (e.g., inert gas condensation), liquid phase (e.g., electrodeposition, rapid solidification), and solid state (e.g., mechanical attrition). In-situ synthesis methods have been used to produce composites. For example, $\mathrm{TiC}$ or $\mathrm{TiB}_{2}$ can be produced by in-situ reaction to reinforce titanium matrix composites. ${ }^{1,2)}$ Premkumar, et al. ${ }^{3)}$ synthesized TiC particles from pure Ti and $\mathrm{C}$ powder in molten $\mathrm{Al}$ to reinforce $\mathrm{Al}$ matrix material. Based on this method, a new method is developed. Molten salts are used as a substitute for the molten $\mathrm{Al}$, and then the powder reactants come to react in the molten salts. Here, the salts are the medium for synthesizing products. Because the salts do not take part in the chemical reaction, and they can dissolve in water, the powder products are easy to collect by filtration.

\section{Experimental}

Ni powder (200 meshes, 99.9\% purity), Ti powder (200 meshes, $99.9 \%$ purity), $\mathrm{NH}_{4} \mathrm{Cl}(99.5 \%$ purity) and carbon powder were used as the raw materials. The reactant powders were mixed for 1 hour in polypropylene containers with milling media of agate balls. The weight ratio of the balls to the powders was 20:1, and the rotary speed of the container was $150 \mathrm{r} / \mathrm{min}$. The container was backfilled with argon to avoid oxidation. Then the mixtures of reactants and equalmolar $\mathrm{NaCl}+\mathrm{KCl}$ were mixed for $1 \mathrm{~h}$.

The reactant powders and salts were pressed into green blocks $15 \mathrm{~mm}$ in diameter and 3 to $5 \mathrm{~mm}$ in thickness by $3 \mathrm{MPa}$ of axial pressure. The green blocks were put into a crucible filled with molten salts $(\mathrm{NaCl}+\mathrm{KCl})$ in a furnace at different temperatures. After a holding period of ten minutes, the crucibles were taken out of the furnace and cooled in air. Finally, the salts containing the product powders were put into distilled water and the salts dissolved. Then the product powders were collected by filtration. It is worth noting that the density of the green blocks is higher than that of the molten salts. Thus, the green blocks sinks down to the bottom of the crucible as soon as it is put into the molten salts, which prevents oxidation during the reaction process.

A Netzsch 204F1 differential scanning calorimeter (DSC) was used to measure the reverse martensitic transformation of the NiTi particles at a heating and cooling rate of $10^{\circ} \mathrm{C} /$ min. The phase composition was analyzed by using the X-ray diffraction (XRD) method with $\mathrm{Cu}-\mathrm{K} \alpha$ radiation.

\section{Results and Discussion}

The TiC/NiTi composite particles were synthesized from the $\mathrm{Ni}, \mathrm{Ti}$ and $\mathrm{C}$ powders by chemical reaction in molten salts. Figure 1 shows the XRD results of the as-prepared TiC/NiTi composite particles. The results indicated the presence of TiC and NiTi alloy in the martensitic phase. The broadening of the diffraction peaks indicated the tiny size of the grains. According to the Scherrer formula, ${ }^{4)}$ the average crystallite size of the $\mathrm{TiC}$ and NiTi particles were 28 and $33 \mathrm{~nm}$, respectively.

Figure 2 shows the DSC curves of the TiC/NiTi composite particles. As can be seen, the reverse transformation starting temperature $\left(A_{\mathrm{s}}\right)$ and the finishing temperature $\left(A_{\mathrm{f}}\right)$ were 60.5 and $104.5^{\circ} \mathrm{C}$, respectively, and in cooling the martensitic starting temperature $\left(M_{\mathrm{S}}\right)$ and finishing temperature $\left(M_{\mathrm{f}}\right)$ were 72.5 and $39.5^{\circ} \mathrm{C}$, respectively. Figure 2

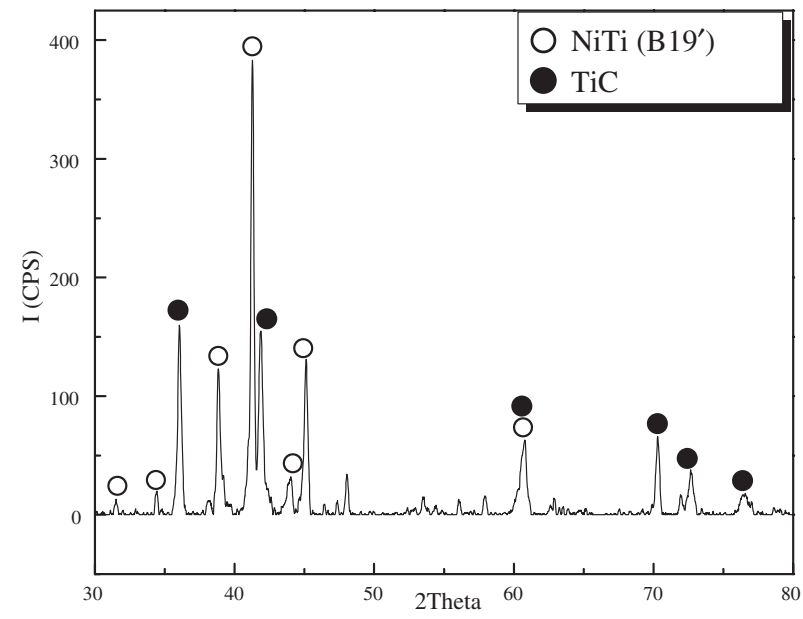

Fig. 1 XRD pattern of TiC/NiTi composite particles. 


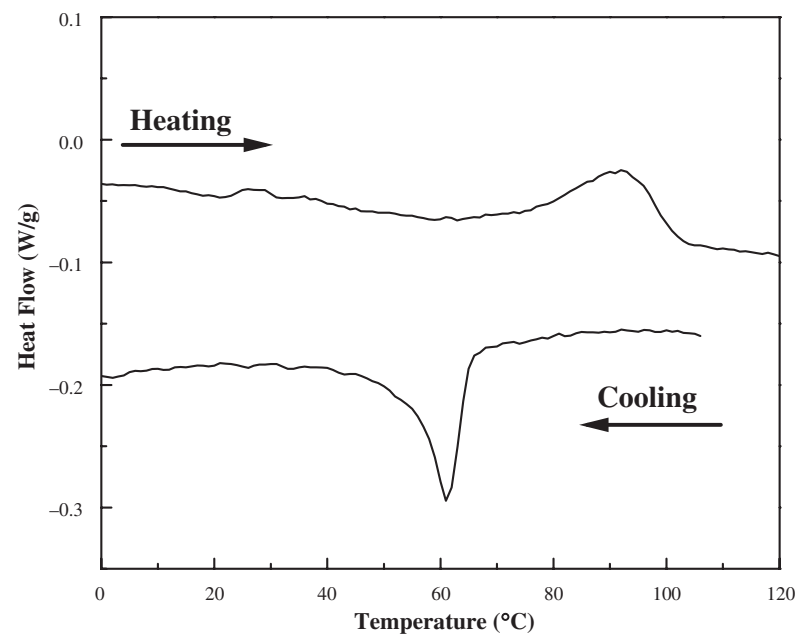

Fig. 2 DSC curves illustrating the reverse martensitic transformation of NiTi particles in the composite particles.

clearly indicates that the prepared NiTi particles possess reversible martensitic transformation characteristics.

In our early researches, $\mathrm{NiTi}$ and $\mathrm{TiC}$ particles were successfully synthesized separately by the high temperature molten salts method. ${ }^{5,6)}$ The reactions between $\mathrm{Ti}$ and $\mathrm{C}$, and $\mathrm{Ti}$ and $\mathrm{Ni}$ are both spontaneous at room temperature, according to eq. (1) and eq. (2),

$$
\begin{array}{ll}
\mathrm{Ni}+\mathrm{Ti}=\mathrm{NiTi} & \Delta G_{298}=-66.462 \mathrm{KJ} / \mathrm{mol} \\
\mathrm{Ti}+\mathrm{C}=\mathrm{TiC} & \Delta G_{298}=-183.92 \mathrm{KJ} / \mathrm{mol}
\end{array}
$$

However, due to the kinetics reasons, the NiTi particles could only be synthesized in molten salts at temperatures not lower than $680^{\circ} \mathrm{C},{ }^{5)}$ and the $\mathrm{TiC}$ particles could only be synthesized in molten salts at temperatures not lower than $800^{\circ} \mathrm{C}$. ${ }^{6)}$ In this work, however, the $\mathrm{TiC} / \mathrm{NiTi}$ composite particles can be synthesized at a temperature lower than $800^{\circ} \mathrm{C}$. It is well known that $\mathrm{Ni}, \mathrm{Ti}$ and $\mathrm{C}$ particles are not soluble in the molten salts. When the green blocks are put into the molten salts, the salts in the green blocks will melt under the circumstance temperature. In the molten salts, if $\mathrm{Ni}$ and $\mathrm{Ti}$ particles meet each other, reactions can happen and NiTi particles can be obtained. According to the eq. (1), the reaction between $\mathrm{Ni}$ and $\mathrm{Ti}$ is exothermic. The released heat elevates the local temperature, which facilitates the reaction between the remaining $\mathrm{Ti}$ and $\mathrm{C}$ particles. This is the reason that the $\mathrm{TiC}$ particles can be synthesized at a temperature lower than $800^{\circ} \mathrm{C}$.

In our early researches, the $\mathrm{TiCN}$ particles were synthesized from the $\mathrm{Ti}, \mathrm{C}$ and $\mathrm{NH}_{4} \mathrm{Cl}$ powders by chemical reaction in molten salts. On the basis of this research, TiCN/ $\mathrm{NiTi}$ composite particles were prepared. In order to attain TiCN/NiTi composite particles, the $\mathrm{Ni}, \mathrm{Ti}, \mathrm{C}$ and $\mathrm{NH}_{4} \mathrm{Cl}$ powders were employed as the raw materials. Figure 3 shows the XRD results of the as-prepared $\mathrm{TiCN} / \mathrm{NiTi}$ composite particles. The results indicated the presence of NiTi alloy in the martensitic phase and TiCN phase. Besides the desired TiCN and NiTi phase, there were some $\mathrm{Ni}_{3} \mathrm{Ti}$ existing. This indicated that the chemical reaction in the molten salts was complicated. Further researches, such as improving the process parameters, should be carried out to reveal the real

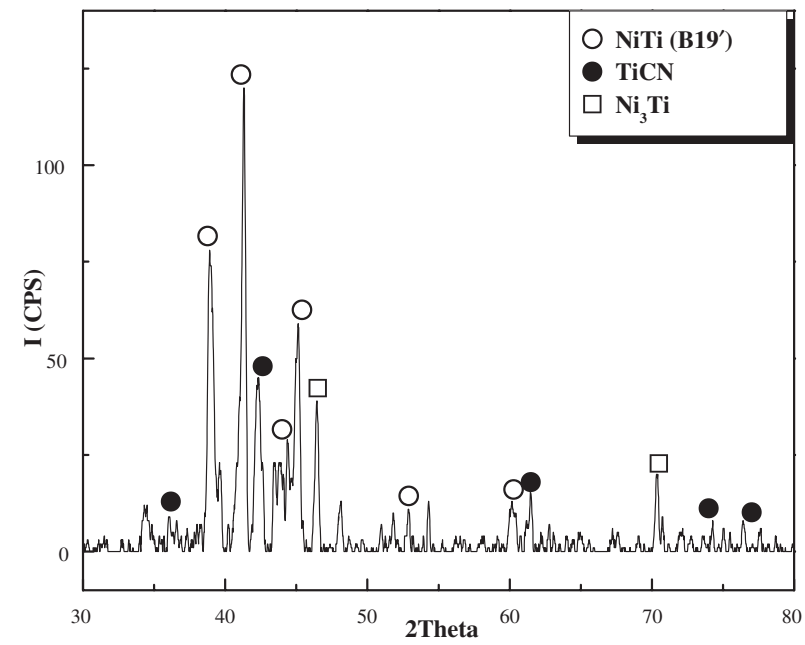

Fig. 3 XRD pattern of TiCN/NiTi composite particles.

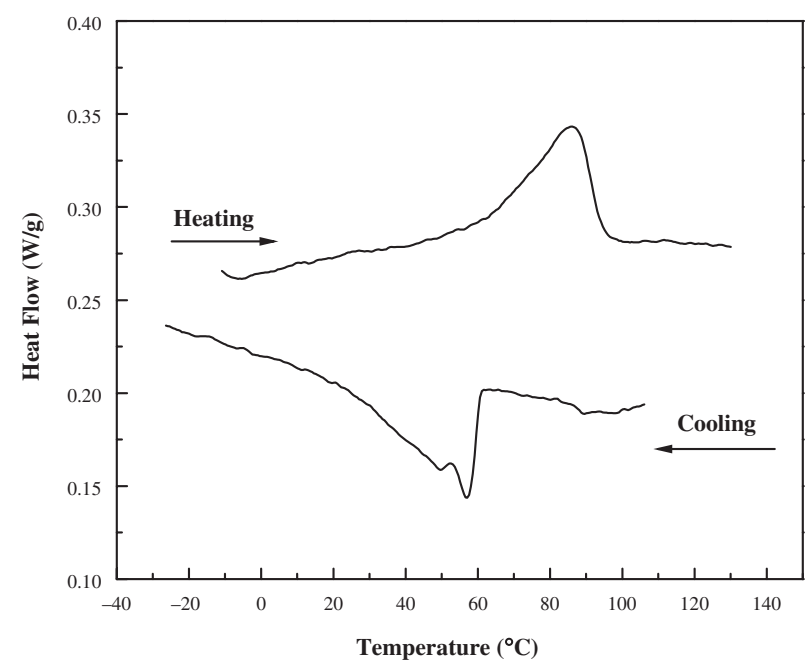

Fig. 4 DSC curves illustrating the reverse martensitic transformation of NiTi particles in the TiCN/NiTi composite particles.

reaction mechanism in the molten salts.

Figure 4 shows the DSC curves of the TiCN/NiTi composite particles. As can be seen, the reverse transformation starting temperature $\left(A_{\mathrm{s}}\right)$ and the finishing temperature $\left(A_{\mathrm{f}}\right)$ were 66.7 and $94.4^{\circ} \mathrm{C}$, respectively, and when cooling the martensitic starting temperature $\left(M_{\mathrm{s}}\right)$ and finishing temperature $\left(M_{\mathrm{f}}\right)$ were 60.5 and $24.5^{\circ} \mathrm{C}$, respectively. Figure 4 clearly indicates that the prepared NiTi particles possess reversible martensitic transformation characteristics.

\section{Conclusion}

TiC/NiTi composite particles are prepared by the high temperature molten salts method. The DSC analysis confirms the presence of the martensite transformation in these particles. The heat of the reaction between $\mathrm{Ni}$ and $\mathrm{Ti}$ could promote the reaction between $\mathrm{Ti}$ and $\mathrm{C}$.

TiCN/NiTi composite particles are prepared by the same method, too. Although the products are complicated, the presence of the martensite transformation in these particles is also confirmed by DSC analysis. 


\section{Acknowledgments}

This work has been supported by the National Natural Science Foundation of China (No. 50271044) and Beijing Natural Science Foundation (No. 3053018).

\section{REFERENCES}

1) W. J. Lu, D. Zhang, X. N. Zhang, R. J. Wu, T. Sakata and H. Mori: J. Alloys Compd. 327 (2001) 248-252.
2) E. L. Zhang, S. Y. Zeng and B. Wang: J. Mater. Process Tech. 125 (2002) 103-109.

3) M. K. Premkumar and M. G. Chu: Mater. Sci. Eng. A-Struct. 202 (1995) 172-178.

4) H. P. Klug and L. E. Alexander: X-Ray diffraction procedures: for polycrystalline and amorphous materials, (John Wiley \& Sons Inc., London, 1974) pp. 491.

5) J. L. Zhao, L. S. Cui, W. F. Gao and Y. J. Zheng: Intermetallics 13 (2005) 301-303.

6) X. L. Cui and L. S. Cui: Key Eng. Mater. 280-283 (2005) 581-586. 